\title{
Existence, non-existence and multiplicity results for a third order eigenvalue three-point boundary value problem
}

\author{
Alberto Cabada ${ }^{\mathrm{a}, *}$, Lucía López-Somozaa ${ }^{\mathrm{a}}$, Feliz Minhós \\ a Instituto de Matemáticas, Facultade de Matemáticas, Universidade de Santiago de Compostela, 15782 Santiago de Compostela, \\ Galicia, Spain. \\ ${ }^{b}$ Departamento de Matemática, Escola de Ciências e Tecnologia, Universidade de Évora, Évora, Portugal. \\ ${ }^{c}$ Centro de Investigação em Matemática e Aplicações (CIMA), Instituto de Investigação e Formação Avançada, Universidade de Évora, \\ Évora, Portugal.
}

\begin{abstract}
This paper provides sufficient conditions to guarantee the existence, non-existence and multiplicity of solutions for a third order eigenvalue fully differential equation, coupled with three point boundary value conditions. Although the change of sign, some bounds for the second derivative of the Green's function are obtained, which allow to define a different kind of cone that, as far as we know, has not been previously used in the literature. The main arguments are based on the fixed point index theory for bounded and unbounded sets. Some examples are presented in order to show that the different existence theorems proved are not comparable. (C)2017 All rights reserved.
\end{abstract}

Keywords: Nonlinear boundary value problems, parameter dependence, multipoint boundary value problems, Green functions, degree theory, fixed points in cones.

2010 MSC: 34B08, 34B10, 34B15, 34B18, 34B27.

\section{Introduction}

In this work we study the existence of solution of the third order nonlinear differential equation

$$
-u^{(3)}(t)=\lambda f\left(t, u(t), u^{\prime}(t), u^{\prime \prime}(t)\right), \quad t \in[0,1],
$$

with $\lambda>0$ a parameter and $f:[0,1] \times \mathbb{R}^{3} \rightarrow[0, \infty)$ an $\mathrm{L}^{1}$-Carathéodory function, coupled with the three point boundary value conditions

$$
u(0)=u^{\prime}(0)=0, \quad u^{\prime}(1)=\alpha u^{\prime}(\eta),
$$

where $0<\eta<1$ and $1<\alpha<\frac{1}{\eta}$ are given constants. Moreover, sufficient conditions for non-existence and multiplicity of solutions are given.

\footnotetext{
${ }^{*}$ Corresponding author

Email addresses: alberto. cabada@usc.es (Alberto Cabada), lucia.lopez.somoza@usc.es (Lucía López-Somoza), fminhos@sapo.pt (Feliz Minhós)

doi:10.22436/jnsa.010.10.28
} 
This type of third order three-point boundary value problems can be seen as a particular case of multipoint problems (as in [12]), nonlocal problems (see [9]), functional problems (as in [3]), or integral equations (see [4]). Therefore all the applications for the above type of problems hold for our problem. More precisely, these third order three-point boundary value problems arise in several areas of applied mathematics and physics, such as the deflection of a curved beam with a constant or varying cross section, three layer beams, electromagnetic waves, gravity driven flows, study of the equilibrium states of a heated bar, and other ones contained in [6].

A precedent problem

$$
\begin{aligned}
& u^{(3)}(t)+a(t) f(u(t))=0, \quad t \in[0,1], \\
& u(0)=u^{\prime}(0)=0, \quad u^{\prime}(1)=\alpha u^{\prime}(\eta),
\end{aligned}
$$

was considered in [8]. There, the authors constructed the Green's function related to the problem and established some of their properties. From them, they built a suitable cone and applied Guo-Krasnoselskii Theorem to assure the existence of a positive solution of the problem.

Recently, in [13], the authors considered the following system

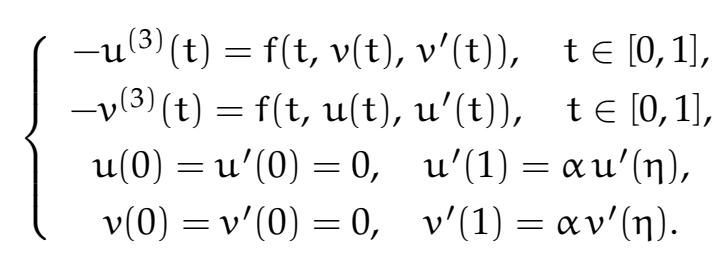

They studied the properties of the first derivative of the Green's function related to the problem and used them to construct a cone $\mathrm{K}$ such that there exist $u, v \in \mathrm{K}$ which constitute a positive solution of the system. To do this, they also used Guo-Krasnoselskii Theorem [7].

In this paper, we study a generalization of the previous equations by considering that the nonlinearity $f$ depends on the solution and its first and second order derivatives. Because of this, we need to examine the properties of the second derivative of the Green's function. However, as we will see along the paper, contrarily to what happened with the Green's function $G$ and its first derivative, in this case it is not possible to find a function $\Phi$ such that

$$
\left|\frac{\partial^{2} \mathrm{G}}{\partial \mathrm{t}^{2}}(\mathrm{t}, \mathrm{s})\right| \leqslant \Phi(\mathrm{s}), \quad \forall(\mathrm{t}, \mathrm{s}) \in[0,1] \times[0,1],
$$

and

$$
\frac{\partial^{2} \mathrm{G}}{\partial \mathrm{t}^{2}}(\mathrm{t}, \mathrm{s}) \geqslant \mathrm{c} \Phi(\mathrm{s}), \quad \forall(\mathrm{t}, \mathrm{s}) \in[\mathrm{a}, \mathrm{b}] \times[0,1]
$$

for some $[a, b] \subset[0,1]$ and $c \in(0,1)$.

This makes it necessary to construct a different kind of cone that, as far as we know, has not been previously used in the literature. With this cone, we will give some conditions to assure the existence of a positive and increasing solution to problem (1.1)-(1.2), which will also be convex in a certain subset of its interval of definition.

The paper is organized in the following way: In Section 2 we compile the known properties for the Green's function related to the problem and its first derivative, and we study its second one. In Section 3 we define our cone and give some existence results by means of the fixed point index for unbounded sets (see [5]). In Section 4, we consider the fixed point index theory for bounded sets in order to obtain some results regarding existence and multiplicity of solutions. We follow the line of results given in $[2,10,11]$. In Section 5, we give some conditions under which there is not any solution for the considered problem. Finally, in Section 6, we give some examples which show that the existence results obtained in Sections 3 and 4 are not comparable. 


\section{Preliminary results}

The Green's function related to the homogeneous problem

$$
\begin{aligned}
& -u^{(3)}(t)=0, \quad t \in[0,1], \\
& u(0)=u^{\prime}(0)=0, \quad u^{\prime}(1)=\alpha u^{\prime}(\eta),
\end{aligned}
$$

is given by the following expression ([8])

$$
G(t, s)=\frac{1}{2(1-\alpha \eta)} \begin{cases}\left(2 t s-s^{2}\right)(1-\alpha \eta)+t^{2} s(\alpha-1), & s \leqslant \min \{\eta, t\} \\ t^{2}(1-\alpha \eta)+t^{2} s(\alpha-1), & t \leqslant s \leqslant \eta \\ \left(2 t s-s^{2}\right)(1-\alpha \eta)+t^{2}(\alpha \eta-s), & \eta \leqslant s \leqslant t \\ t^{2}(1-s), & \max \{\eta, t\} \leqslant s\end{cases}
$$

Next lemmas establish some properties of the Green's function and its first and second order derivatives.

Lemma 2.1 ([8, Lemma 2.2]). Let $0<\eta<1$ and $1<\alpha<\frac{1}{\eta}$. Then,

$$
0 \leqslant G(t, s) \leqslant g_{0}(s)=\frac{1+\alpha}{1-\alpha \eta} s(1-s), \quad \forall(t, s) \in[0,1] \times[0,1] .
$$

Lemma 2.2 ([8, Lemma 2.3]). Let $0<\eta<1$ and $1<\alpha<\frac{1}{\eta}$. Then,

$$
\mathrm{G}(\mathrm{t}, \mathrm{s}) \geqslant \mathrm{k}_{0} \mathrm{~g}_{0}(\mathrm{~s}), \quad \forall(\mathrm{t}, \mathrm{s}) \in\left[\frac{\eta}{\alpha}, \eta\right] \times[0,1],
$$

with $0<\kappa_{0}=\frac{\eta^{2}}{2 \alpha^{2}(1+\alpha)} \min \{\alpha-1,1\}<1$.

The first derivative of $G$ is given by

$$
\frac{\partial G}{\partial t}(t, s)=\frac{1}{(1-\alpha \eta)} \begin{cases}s(1-\alpha \eta)+t s(\alpha-1), & s \leqslant \min \{\eta, t\} \\ t(1-\alpha \eta)+t s(\alpha-1), & t \leqslant s \leqslant \eta \\ s(1-\alpha \eta)+t(\alpha \eta-s), & \eta \leqslant s \leqslant t \\ t(1-s), & \max \{\eta, t\} \leqslant s\end{cases}
$$

and satisfies the following properties.

Lemma 2.3 ([13, Lemma 3]). Let $0<\eta<1$ and $1<\alpha<\frac{1}{\eta}$. Then,

$$
0 \leqslant \frac{\partial G}{\partial t}(t, s) \leqslant g_{1}(s)=\frac{1-s}{1-\alpha \eta}, \quad \forall(t, s) \in[0,1] \times[0,1] .
$$

Lemma 2.4 ([13, Lemma 4]). Let $0<\eta<1$ and $1<\alpha<\frac{1}{\eta}$. Then,

$$
\frac{\partial G}{\partial t}(t, s) \geqslant \kappa_{1} g_{1}(s), \quad \forall(t, s) \in\left[\frac{\eta}{\alpha}, \eta\right] \times[0,1],
$$

with $0<\kappa_{1}=\eta<1$.

The second derivative of $\mathrm{G}$ is given by

$$
\frac{\partial^{2} G}{\partial t^{2}}(t, s)=\frac{1}{1-\alpha \eta} \begin{cases}s(\alpha-1), & s \leqslant \min \{\eta, t\} \\ 1-\alpha \eta+s(\alpha-1), & t \leqslant s \leqslant \eta \\ \alpha \eta-s, & \eta \leqslant s \leqslant t \\ 1-s, & \max \{\eta, t\} \leqslant s .\end{cases}
$$

It is immediate to verify that it satisfies the following conditions: 
Lemma 2.5. Let $0<\eta<1$ and $1<\alpha<\frac{1}{\eta}$. Then,

$$
\frac{\partial^{2} G}{\partial t^{2}}(t, s) \geqslant 0, \quad \forall(t, s) \in([0,1] \times[0,1]) \backslash A,
$$

where

$$
A=\{(t, s) \in[0,1] \times[0,1] ; \alpha \eta<t<1, \alpha \eta<s<t\} .
$$

Remark 2.6. Note that, in particular, $\frac{\partial^{2} G}{\partial t^{2}}(t, s) \geqslant 0$ for all $(t, s) \in[0, \alpha \eta] \times[0,1]$.

Next two results will allow us to define a suitable cone in $\mathcal{C}^{2}[0,1]$.

Lemma 2.7. Let $0<\eta<1$ and $1<\alpha<\frac{1}{\eta}$. Then,

$$
\frac{\partial^{2} \mathrm{G}}{\partial \mathrm{t}^{2}}(\mathrm{t}, \mathrm{s}) \leqslant \mathrm{g}_{2}(\mathrm{~s}), \quad \forall(\mathrm{t}, \mathrm{s}) \in\left[\frac{\eta}{\alpha}, 1\right] \times[0,1],
$$

and

$$
-1 \leqslant \frac{\partial^{2} G}{\partial t^{2}}(t, s) \leqslant \max \left\{g_{2}(s), \frac{1-\eta}{1-\alpha \eta}\right\}, \quad \forall(t, s) \in[0,1] \times[0,1],
$$

with

$$
g_{2}(s)=\frac{\alpha(1-\alpha \eta)+\eta(\alpha-1)}{\eta(\alpha-1)} \frac{\partial^{2} G}{\partial t^{2}}(\eta, s)=\frac{\alpha(1-\alpha \eta)+\eta(\alpha-1)}{\eta(\alpha-1)(1-\alpha \eta)} \begin{cases}s(\alpha-1), & 0 \leqslant s \leqslant \eta, \\ 1-s, & \eta \leqslant s \leqslant 1 .\end{cases}
$$

Proof. First, we will prove that $\frac{\partial^{2} G}{\partial t^{2}}(t, s) \leqslant g_{2}(s)$ for all $(t, s) \in\left[\frac{\eta}{\alpha}, 1\right] \times[0,1]$.

For $s \leqslant \min \{\eta, t\}$ and $s \geqslant \max \{\eta, t\}$ we have that $\frac{\partial^{2} G}{\partial t^{2}}(t, s)=\frac{\partial^{2} G}{\partial t^{2}}(\eta, s)$ and since $\frac{\alpha(1-\alpha \eta)}{\eta(\alpha-1)}+1>1$, it is obvious that $\frac{\partial^{2} G}{\partial t^{2}}(t, s) \leqslant g_{2}(s)$.

For $t \leqslant s \leqslant \eta$, we have that

$$
\frac{\partial^{2} G}{\partial t^{2}}(t, s)=\frac{1-\alpha \eta+s(\alpha-1)}{1-\alpha \eta}=\frac{(\alpha-1)\left(\frac{\eta \alpha(1-\alpha \eta)}{\alpha \eta(\alpha-1)}+s\right)}{1-\alpha \eta} \leqslant \frac{s(\alpha-1)\left(\frac{\alpha(1-\alpha \eta)}{\eta(\alpha-1)}+1\right)}{1-\alpha \eta}=g_{2}(s) .
$$

Finally, for $\eta \leqslant s \leqslant t$,

$$
\frac{\partial^{2} G}{\partial t^{2}}(t, s)=\frac{\alpha \eta-s}{1-\alpha \eta} \leqslant \frac{1-s}{1-\alpha \eta}=\frac{\partial^{2} G}{\partial t^{2}}(\eta, s) \leqslant g_{2}(s) .
$$

Now, we will prove that

$$
\frac{\partial^{2} G}{\partial t^{2}}(t, s) \geqslant-1 \text { for all }(t, s) \in[0,1] \times[0,1]
$$

It is immediate to verify that $\frac{\partial^{2} G}{\partial t^{2}}(t, s) \geqslant 0$ for $s \leqslant \min \{\eta, t\}, t \leqslant s \leqslant \eta$ and $\max \{\eta, t\} \leqslant s$. On the other hand, for $\eta \leqslant s \leqslant t$, we have that

$$
\frac{\partial^{2} G}{\partial t^{2}}(t, s)=\frac{\alpha \eta-s}{1-\alpha \eta} \geqslant \frac{\alpha \eta-1}{1-\alpha \eta}=-1,
$$

and so the result holds.

Finally, we will prove that

$$
\frac{\partial^{2} G}{\partial t^{2}}(t, s) \leqslant \max \left\{g_{2}(s), \frac{1-\eta}{1-\alpha \eta}\right\}, \quad \forall(t, s) \in[0,1] \times[0,1]
$$


Obviously, it is enough to prove the inequality for $(t, s) \in\left[0, \frac{\eta}{\alpha}\right] \times[0,1]$.

For $s \leqslant t$ and $s \geqslant \eta$, we have just seen that $\frac{\partial^{2} G}{\partial t^{2}}(t, s)=\frac{\partial^{2} G}{\partial t^{2}}(\eta, s) \leqslant g_{2}(s)$.

On the other hand, for $t \leqslant s \leqslant \eta$, the following inequality holds

$$
\frac{\partial^{2} G}{\partial t^{2}}(t, s)=\frac{1-\alpha \eta+s(\alpha-1)}{1-\alpha \eta} \leqslant \frac{1-\alpha \eta+\eta(\alpha-1)}{1-\alpha \eta}=\frac{1-\eta}{1-\alpha \eta}
$$

and so the result is proved.

Remark 2.8. We note that for any constant $c \in(0,1)$ it would be possible to find a continuous function $\mathrm{g} \neq \mathrm{g}_{2}$ such that

$$
\frac{\partial^{2} \mathrm{G}}{\partial \mathrm{t}^{2}}(\mathrm{t}, \mathrm{s}) \leqslant \mathrm{g}(\mathrm{s}), \quad \forall(\mathrm{t}, \mathrm{s}) \in[\mathrm{c}, 1] \times[0,1] .
$$

Lemma 2.9. Let $0<\eta<1$ and $1<\alpha<\frac{1}{\eta}$. Then, for all $(t, s) \in\left[\frac{\eta}{\alpha}, \eta\right] \times[0,1]$,

$$
\frac{\partial^{2} G}{\partial t^{2}}(t, s) \geqslant \frac{\partial^{2} G}{\partial t^{2}}(\eta, s) \equiv \kappa_{2} g_{2}(s) \text {, }
$$

with $0<\kappa_{2}=\frac{\eta(\alpha-1)}{\alpha(1-\alpha \eta)+\eta(\alpha-1)}$.

Proof. For $s \leqslant t$ and $s \geqslant \eta$, we have that $\frac{\partial^{2} G}{\partial t^{2}}(t, s)=\kappa_{2} g_{2}(s)$.

On the other hand, for $t \leqslant s \leqslant \eta$, it holds that

$$
\frac{\partial^{2} G}{\partial t^{2}}(t, s)=\frac{1-\alpha \eta+s(\alpha-1)}{1-\alpha \eta} \geqslant \frac{s(\alpha-1)}{1-\alpha \eta}=\kappa_{2} g_{2}(s)
$$

Remark 2.10. We note that for any interval $[a, b] \subset(0, \alpha \eta)$ it would be possible to find a constant $k$ such that

$$
\frac{\partial^{2} \mathrm{G}}{\partial \mathrm{t}^{2}}(\mathrm{t}, \mathrm{s}) \geqslant \kappa \mathrm{g}_{2}(\mathrm{~s}), \quad \forall(\mathrm{t}, \mathrm{s}) \in[\mathrm{a}, \mathrm{b}] \times[0,1] .
$$

However, for the sake of simplicity, we have chosen the interval $\left[\frac{\eta}{\alpha}, \eta\right]$ to maintain the same interval than in Lemmas 2.2 and 2.4.

Remark 2.11. We point out that, on the contrary to function $G$ and $\frac{\partial G}{\partial t}$, it is not possible to find a continuous function $\tilde{g}_{2}(\mathrm{~s})$ such that

and

$$
\left|\frac{\partial^{2} G}{\partial t^{2}}(t, s)\right| \leqslant \tilde{g}_{2}(s), \quad \forall(t, s) \in[0,1] \times[0,1],
$$

with $[a, b] \subset[0,1]$ and $\tilde{\kappa}_{2} \in(0,1)$.

$$
\frac{\partial^{2} G}{\partial t^{2}}(t, s) \geqslant \tilde{\kappa}_{2} \tilde{g}_{2}(s), \quad \forall(t, s) \in[a, b] \times[0,1],
$$

This is due to the fact that for $s \geqslant \alpha \eta$,

$$
\left|\frac{\partial^{2} G}{\partial t^{2}}(t, s)\right|= \begin{cases}\frac{s-\alpha \eta}{1-\alpha \eta}, & s \leqslant t \\ \frac{1-s}{1-\alpha \eta}, & t \leqslant s\end{cases}
$$

As a consequence, if there exists $\tilde{g}_{2}$ satisfying the previous conditions, it would necessarily satisfy that

$$
\tilde{g}_{2}(s) \geqslant \max \left\{\frac{s-\alpha \eta}{1-\alpha \eta}, \frac{1-s}{1-\alpha \eta}\right\}= \begin{cases}\frac{1-s}{1-\alpha \eta}, & s \leqslant \frac{1+\alpha \eta}{2}, \\ \frac{s-\alpha \eta}{1-\alpha \eta}, & s \geqslant \frac{1+\alpha \eta}{2},\end{cases}
$$

for $s \geqslant \alpha \eta$, and so $\tilde{g}_{2}(1) \geqslant 1$.

On the other hand, we have that $\frac{\partial^{2} G}{\partial t^{2}}(t, 1)=0$, so if there exists $\tilde{g}_{2}$ in the previous conditions, it would happen that

which is a contradiction.

$$
0=\frac{\partial^{2} G}{\partial t^{2}}(t, 1) \geqslant \tilde{\kappa}_{2} \tilde{g}_{2}(1) \geqslant \tilde{\kappa}_{2}>0,
$$




\section{Main results}

Let us consider $E=\mathcal{C}^{2}([0,1], \mathbb{R})$ equipped with the norm

$$
\|u\|=\max \left\{\|u\|_{\infty},\left\|u^{\prime}\right\|_{\infty},\left\|u^{\prime \prime}\right\|_{\infty}\right\},
$$

where $\|v\|_{\infty}=\sup _{t \in[0,1]}|v(t)|$. It is very well-known that $(E,\|\cdot\|)$ is a Banach space.

Taking into account the properties satisfied by the Green's function and its derivatives, we define

$$
\begin{array}{r}
K=\left\{u \in \mathcal{C}^{2}([0,1], \mathbb{R}): u(t) \geqslant 0, t \in[0,1], u^{\prime}(t) \geqslant 0, t \in[0,1], u^{\prime \prime}(t) \geqslant 0, t \in[0, \alpha \eta],\right. \\
\left.\min _{t \in\left[\frac{\eta}{\alpha}, \eta\right]} u(t) \geqslant k_{0}\|u\|_{\infty}, \min _{t \in\left[\frac{\eta}{\alpha}, \eta\right]} u^{\prime}(t) \geqslant k_{1}\left\|u^{\prime}\right\|_{\infty}, \min _{t \in\left[\frac{\eta}{\alpha}, \eta\right]} u^{\prime \prime}(t) \geqslant k_{2}\left\|u^{\prime \prime}\right\|_{\left[\frac{\eta}{\alpha}, \eta\right]}\right\},
\end{array}
$$

where

$$
\left\|\mathfrak{u}^{\prime \prime}\right\|_{\left[\frac{\mathfrak{\eta}}{\alpha}, \eta\right]}:=\max _{\mathfrak{t} \in\left[\frac{\mathfrak{\eta}}{\alpha}, \eta\right]}\left|\mathfrak{u}^{\prime \prime}(\mathfrak{t})\right|^{\prime}
$$

and $\kappa_{0}, \kappa_{1}$ and $\kappa_{2}$ are defined in previous section.

It is obvious that $\mathrm{K}$ is a cone in $\mathrm{E}$.

Moreover, it is very well-known that the solutions of the problem (1.1)-(1.2) correspond with the fixed points of the integral operator

$$
\mathrm{Tu}(\mathrm{t})=\lambda \int_{0}^{1} \mathrm{G}(\mathrm{t}, \mathrm{s}) \mathrm{f}\left(\mathrm{s}, \mathrm{u}(\mathrm{s}), \mathrm{u}^{\prime}(\mathrm{s}), \mathrm{u}^{\prime \prime}(\mathrm{s})\right) \mathrm{ds}, \quad \mathrm{t} \in[0,1] .
$$

We make the following assumptions on the elements that take part in the previous expression:

(H1) $\lambda$ is a positive parameter.

(H2) The nonlinearity $f:[0,1] \times \mathbb{R}^{3} \rightarrow[0, \infty)$ satisfies $L^{1}$-Carathéodory conditions, that is,

- $f(\cdot, u, v, w)$ is measurable for each $(u, v, w)$ fixed.

- $f(t, \cdot, \cdot, \cdot)$ is continuous for a.e. $t \in[0,1]$.

- For each $r>0$ there exists $\phi_{\mathrm{r}} \in \mathrm{L}^{1}[0,1]$ such that

$$
f(t, u, v, w) \leqslant \phi_{r}(t), \quad \forall(u, v, w) \in(-r, r) \times(-r, r) \times(-r, r), \text { a.e. } t \in[0,1]
$$

Under these assumptions, coupled with some additional properties on the function $f$, we will ensure the existence of solutions of the considered problem (1.1)-(1.2). Before doing that, we will obtain some previous technical results.

Lemma 3.1. $\mathrm{T}: \mathrm{K} \rightarrow \mathrm{K}$ is a completely continuous operator.

Proof. We divide the proof into several steps.

Step 1. T is well-defined in $\mathrm{K}$.

Let $u \in K$. We will prove that $T u \in K$.

It is obvious that $\mathrm{Tu}(\mathrm{t}) \geqslant 0$ for all $t \in[0, T]$. Moreover, using Lemma 2.1, we have

$$
T u(t)=\lambda \int_{0}^{1} G(t, s) f\left(s, u(s), u^{\prime}(s), u^{\prime \prime}(s)\right) d s \leqslant \lambda \int_{0}^{1} g_{0}(s) f\left(s, u(s), u^{\prime}(s), u^{\prime \prime}(s)\right) d s,
$$

and, taking the supremum for $t \in[0,1]$, we deduce that

$$
\|T u\|_{\infty} \leqslant \lambda \int_{0}^{1} g_{0}(s) f\left(s, u(s), u^{\prime}(s), u^{\prime \prime}(s)\right) d s .
$$


So, for $t \in\left[\frac{\eta}{\alpha}, \eta\right]$, from Lemma 2.2, we have

$$
\begin{aligned}
T u(t) & =\lambda \int_{0}^{1} G(t, s) f\left(s, u(s), u^{\prime}(s), u^{\prime \prime}(s)\right) d s \\
& \geqslant \lambda \int_{0}^{1} k_{0} g_{0}(s) f\left(s, u(s), u^{\prime}(s), u^{\prime \prime}(s)\right) d s \geqslant k_{0}\|T u\|_{\infty},
\end{aligned}
$$

and we deduce that

$$
\min _{t \in\left[\frac{\eta}{\alpha}, \eta\right]} T u(t) \geqslant \kappa_{0}\|T u\|_{\infty}
$$

Analogously, since $\frac{\partial G}{\partial t}(t, s) \geqslant 0$ on $[0,1] \times[0,1]$, it is immediate to verify that

$$
(T u)^{\prime}(t)=\lambda \int_{0}^{1} \frac{\partial G}{\partial t}(t, s) f\left(s, u(s), u^{\prime}(s), u^{\prime \prime}(s)\right) d s \geqslant 0 .
$$

Moreover, Lemma 2.3 implies

$$
(T u)^{\prime}(t) \leqslant \lambda \int_{0}^{1} g_{1}(s) f\left(s, u(s), u^{\prime}(s), u^{\prime \prime}(s)\right) d s,
$$

and, taking the supremum for $t \in[0,1]$,

$$
\left\|(T u)^{\prime}\right\|_{\infty} \leqslant \lambda \int_{0}^{1} g_{1}(s) f\left(s, u(s), u^{\prime}(s), u^{\prime \prime}(s)\right) d s .
$$

So, for $t \in\left[\frac{\eta}{\alpha}, \eta\right]$, Lemma 2.4 gives us

$$
(T u)^{\prime}(t) \geqslant \lambda \int_{0}^{1} k_{1} g_{1}(s) f\left(s, u(s), u^{\prime}(s), u^{\prime \prime}(s)\right) d s \geqslant k_{1}\left\|(T u)^{\prime}\right\|_{\infty}
$$

and we can affirm that

$$
\min _{t \in\left[\frac{\eta}{\alpha}, \eta\right]}(T u)^{\prime}(t) \geqslant k_{1}\left\|(T u)^{\prime}\right\|_{\infty} .
$$

Finally, from Lemma 2.5, we have that for $t \in[0, \alpha \eta]$,

$$
(T u)^{\prime \prime}(t)=\lambda \int_{0}^{1} \frac{\partial^{2} G}{\partial t^{2}}(t, s) f\left(s, u(s), u^{\prime}(s), u^{\prime \prime}(s)\right) d s \geqslant 0 .
$$

In addition, for $t \in\left[\frac{\eta}{\alpha}, \eta\right]$, Lemma 2.7 assures that

$$
(T u)^{\prime \prime}(t) \leqslant \lambda \int_{0}^{1} g_{2}(s) f\left(s, u(s), u^{\prime}(s), u^{\prime \prime}(s)\right) d s,
$$

and, taking the supremum for $t \in\left[\frac{\eta}{\alpha}, \eta\right]$,

$$
\left\|(T u)^{\prime \prime}\right\|_{\left[\frac{\eta}{\alpha}, \eta\right]} \leqslant \lambda \int_{0}^{1} g_{2}(s) f\left(s, u(s), u^{\prime}(s), u^{\prime \prime}(s)\right) d s .
$$

So, for $t \in\left[\frac{\eta}{\alpha}, \eta\right]$, from Lemma 2.9 we know that

$$
(T u)^{\prime \prime}(t) \geqslant \lambda \int_{0}^{1} k_{2} g_{2}(s) f\left(s, u(s), u^{\prime}(s), u^{\prime \prime}(s)\right) d s \geqslant k_{2}\left\|(T u)^{\prime \prime}\right\|_{\left[\frac{\eta}{\alpha}, \eta\right]}{ }^{\prime}
$$

and we deduce that

$$
\min _{t \in\left[\frac{\eta}{\alpha}, \eta\right]}(T u)^{\prime \prime}(t) \geqslant \kappa_{2}\left\|(T u)^{\prime}\right\|_{\left[\frac{\eta}{\alpha}, \eta\right]} .
$$

Therefore, we can conclude that $T u \in K$. 
Step 2. T is a compact operator.

Let us consider

$$
\mathrm{B}=\{\mathrm{u} \in \mathrm{E} ;\|\mathrm{u}\| \leqslant \mathrm{r}\} .
$$

First, we will prove that $T(B)$ is uniformly bounded in $\mathcal{C}^{2}[0,1]$. We find the following bounds for $u \in B$ :

$$
\begin{aligned}
\|T u\|_{\infty} & =\sup _{t \in[0,1]}\left|\lambda \int_{0}^{1} G(t, s) f\left(s, u(s), u^{\prime}(s), u^{\prime \prime}(s)\right) d s\right| \\
& \leqslant \lambda \int_{0}^{1} g_{0}(s) f\left(s, u(s), u^{\prime}(s), u^{\prime \prime}(s)\right) d s \leqslant \lambda \int_{0}^{1} g_{0}(s) \phi_{r}(s) d s:=M_{1} . \\
\left\|(T u)^{\prime}\right\|_{\infty} & =\sup _{t \in[0,1]}\left|\lambda \int_{0}^{1} \frac{\partial G}{\partial t}(t, s) f\left(s, u(s), u^{\prime}(s), u^{\prime \prime}(s)\right) d s\right| \\
& \leqslant \lambda \int_{0}^{1} g_{1}(s) f\left(s, u(s), u^{\prime}(s), u^{\prime \prime}(s)\right) d s \leqslant \lambda \int_{0}^{1} g_{1}(s) \phi_{r}(s) d s:=M_{2} . \\
\left\|(T u)^{\prime \prime}\right\|_{\infty} & =\sup _{t \in[0,1]}\left|\lambda \int_{0}^{1} \frac{\partial^{2} G}{\partial t^{2}}(t, s) f\left(s, u(s), u^{\prime}(s), u^{\prime \prime}(s)\right) d s\right| \\
& \leqslant \sup _{t \in[0,1]} \lambda \int_{0}^{1}\left|\frac{\partial^{2} G}{\partial t^{2}}(t, s)\right| f\left(s, u(s), u^{\prime}(s), u^{\prime \prime}(s)\right) d s \\
& \leqslant \lambda \int_{0}^{1} \max \left\{\frac{1-\eta}{1-\alpha \eta}, g_{2}(s)\right\} f\left(s, u(s), u^{\prime}(s), u^{\prime \prime}(s)\right) d s \\
& \leqslant \lambda \int_{0}^{1} \max \left\{\frac{1-\eta}{1-\alpha \eta}, g_{2}(s)\right\} \phi_{r}(s) d s:=M_{3} .
\end{aligned}
$$

So, it is deduced that

$$
\|T u\| \leqslant \max \left\{M_{1}, M_{2}, M_{3}\right\}, \quad \forall u \in B .
$$

Now, we will prove that $T(B)$ is equicontinuous in $\mathrm{C}^{2}[0,1]$.

Let $t_{1}, t_{2} \in[0,1]$. Without loss of generality, suppose that $t_{1}<t_{2}$. Then,

$$
\begin{aligned}
\left|T u\left(t_{1}\right)-T u\left(t_{2}\right)\right| & \leqslant \lambda \int_{0}^{1}\left|G\left(t_{1}, s\right)-G\left(t_{2}, s\right)\right| f\left(s, u(s), u^{\prime}(s), u^{\prime \prime}(s)\right) d s \\
& \leqslant \lambda \int_{0}^{1}\left|G\left(t_{1}, s\right)-G\left(t_{2}, s\right)\right| \phi_{r}(s) d s
\end{aligned}
$$

and since $G(\cdot, s)$ is continuous, we have that for all $\varepsilon>0$ there exists $\delta(\varepsilon)>0$ such that if $\left|t_{2}-t_{1}\right|<\delta$ then $\left|T u\left(t_{1}\right)-T u\left(t_{2}\right)\right|<\varepsilon$ for all $u \in B$.

Analogously,

$$
\begin{aligned}
\left|(T u)^{\prime}\left(t_{1}\right)-(T u)^{\prime}\left(t_{2}\right)\right| & \leqslant \lambda \int_{0}^{1}\left|\frac{\partial G}{\partial t}\left(t_{1}, s\right)-\frac{\partial G}{\partial t}\left(t_{2}, s\right)\right| f\left(s, u(s), u^{\prime}(s), u^{\prime \prime}(s)\right) d s \\
& \leqslant \lambda \int_{0}^{1}\left|\frac{\partial G}{\partial t}\left(t_{1}, s\right)-\frac{\partial G}{\partial t}\left(t_{2}, s\right)\right| \phi_{r}(s) d s
\end{aligned}
$$

and, since $\frac{\partial G}{\partial t}(\cdot, s)$ is also continuous, we reason as in the previous case and conclude that

$$
\left|(T u)^{\prime}\left(t_{1}\right)-(T u)^{\prime}\left(t_{2}\right)\right|<\varepsilon, \quad \forall u \in B .
$$


Finally, we have that

$$
\begin{aligned}
\left|(T u)^{\prime \prime}\left(t_{1}\right)-(T u)^{\prime \prime}\left(t_{2}\right)\right| \leqslant & \lambda \int_{0}^{1}\left|\frac{\partial^{2} G}{\partial t^{2}}\left(t_{1}, s\right)-\frac{\partial^{2} G}{\partial t^{2}}\left(t_{2}, s\right)\right| f\left(s, u(s), u^{\prime}(s), u^{\prime \prime}(s)\right) d s \\
\leqslant & \lambda \int_{0}^{1}\left|\frac{\partial^{2} G}{\partial t^{2}}\left(t_{1}, s\right)-\frac{\partial^{2} G}{\partial t^{2}}\left(t_{2}, s\right)\right| \phi_{r}(s) d s \\
= & \lambda \int_{0}^{t_{1}}\left|\frac{\partial^{2} G}{\partial t^{2}}\left(t_{1}, s\right)-\frac{\partial^{2} G}{\partial t^{2}}\left(t_{2}, s\right)\right| \phi_{r}(s) d s \\
& +\lambda \int_{t_{1}}^{t_{2}}\left|\frac{\partial^{2} G}{\partial t^{2}}\left(t_{1}, s\right)-\frac{\partial^{2} G}{\partial t^{2}}\left(t_{2}, s\right)\right| \phi_{r}(s) d s \\
& +\lambda \int_{t_{2}}^{1}\left|\frac{\partial^{2} G}{\partial t^{2}}\left(t_{1}, s\right)-\frac{\partial^{2} G}{\partial t^{2}}\left(t_{2}, s\right)\right| \phi_{r}(s) d s
\end{aligned}
$$

In this case, we have that $\frac{\partial^{2} G}{\partial t^{2}}(\cdot, s)$ is continuous in $[0, s) \cup(s, 1]$ and has a jump discontinuity at $t=s$. Because of this, we can apply the same reasoning that in previous cases to assure that the first and last terms in the previous inequality tend to zero with independence of the function $u \in B$. On the other hand, we have that $\left|\frac{\partial^{2} G}{\partial t^{2}}\left(t_{1}, \cdot\right)-\frac{\partial^{2} G}{\partial t^{2}}\left(t_{2}, \cdot\right)\right| \phi_{r}(\cdot) \in L^{1}[0,1]$ so it is obvious that

$$
\lambda \int_{t_{1}}^{t_{2}}\left|\frac{\partial^{2} G}{\partial t^{2}}\left(t_{1}, s\right)-\frac{\partial^{2} G}{\partial t^{2}}\left(t_{2}, s\right)\right| \phi_{r}(s) d s \underset{t_{1} \rightarrow t_{2}}{\longrightarrow} 0,
$$

with independence of the function $u \in B$.

Therefore we conclude that $\mathrm{T}(\mathrm{B})$ is equicontinuous in $\mathrm{C}^{2}[0,1]$.

As a consequence, by Ascoli-Arzelà Theorem, we can affirm that $\mathrm{T}(\mathrm{B})$ is relatively compact in $\mathcal{C}^{2}[0,1]$ and so $\mathrm{T}$ is a completely continuous operator.

We introduce now the following notation (see Remark 3.4 for details)

$$
\begin{gathered}
\Lambda_{1}=\int_{0}^{1} g_{0}(s) d s, \quad \Lambda_{2}=\int_{0}^{1} g_{1}(s) d s, \quad \Lambda_{3}=\int_{0}^{1} \max \left\{g_{2}(s), \frac{1-\eta}{1-\alpha \eta}\right\} d s, \\
\Lambda_{4}=\int_{\frac{\eta}{\alpha}}^{\eta} \kappa_{0} g_{0}(s) d s, \quad \Lambda_{5}=\int_{\frac{\eta}{\alpha}}^{\eta} \kappa_{1} g_{1}(s) d s,
\end{gathered}
$$

and we define

$$
\bar{\Lambda}=3 \max \left\{\Lambda_{1}, \Lambda_{2}, \Lambda_{3}\right\} \text { and } \underline{\Lambda}=\max \left\{\kappa_{0} \Lambda_{4}, \kappa_{1} \Lambda_{5}\right\}
$$

We also denote:

$$
f_{0}=\lim _{|x|,|y|,|z| \rightarrow 0} \min _{t \in[0,1]} \frac{f(t, x, y, z)}{|x|+|y|+|z|}
$$

and

$$
f^{\infty}=\lim _{|x|,|y|,|z| \rightarrow \infty} \max _{t \in[0,1]} \frac{f(t, x, y, z)}{|x|+|y|+|z|} .
$$

We will give now our first existence result.

Theorem 3.2. Assume that hypotheses (H1)-(H2) hold. If $\bar{\Lambda} f^{\infty}<\Lambda f_{0}$, then for all

$$
\lambda \in\left(\frac{1}{\underline{\Lambda} f_{0}}, \frac{1}{\bar{\Lambda} f^{\infty}}\right),
$$

problem (1.1)-(1.2) has at least a positive solution that belongs to the cone $\mathrm{K}$. 
Proof. Let $\lambda \in\left(\frac{1}{\underline{\Lambda} f_{0}}, \frac{1}{\bar{\Lambda} f^{\infty}}\right)$ and choose $\varepsilon \in\left(0, f_{0}\right)$ such that

$$
\frac{1}{\Lambda\left(f_{0}-\varepsilon\right)} \leqslant \lambda \leqslant \frac{1}{\bar{\Lambda}\left(f^{\infty}+\varepsilon\right)}
$$

Taking into account the definition of $f_{0}$, we know that there exists $\delta_{1}>0$ such that when $\|u\| \leqslant \delta_{1}$,

$$
f\left(t, u(t), u^{\prime}(t), u^{\prime \prime}(t)\right)>\left(f_{0}-\varepsilon\right)\left(|u(t)|+\left|u^{\prime}(t)\right|+\left|u^{\prime \prime}(t)\right|\right), \quad \forall t \in[0,1] .
$$

Let

$$
\Omega_{\delta_{1}}=\left\{u \in K ;\|u\|<\delta_{1}\right\},
$$

and choose $u \in \partial \Omega_{\delta_{1}}$. We will prove that $\mathrm{Tu} \npreceq u$. We have that

$$
\begin{aligned}
T u(t) & =\lambda \int_{0}^{1} G(t, s) f\left(s, u(s), u^{\prime}(s), u^{\prime \prime}(s)\right) d s \geqslant \lambda \int_{0}^{1} \kappa_{0} g_{0}(s) f\left(s, u(s), u^{\prime}(s), u^{\prime \prime}(s)\right) d s \\
& \geqslant \lambda \int_{\frac{\eta}{\alpha}}^{\eta} \kappa_{0} g_{0}(s) f\left(s, u(s), u^{\prime}(s), u^{\prime \prime}(s)\right) d s \\
& >\lambda \int_{\frac{\eta}{\alpha}}^{\eta} \kappa_{0} g_{0}(s)\left(f_{0}-\varepsilon\right)\left(|u(s)|+\left|u^{\prime}(s)\right|+\left|u^{\prime \prime}(s)\right|\right) d s \\
& \geqslant \lambda\left(f_{0}-\varepsilon\right)\left(\kappa_{0}\|u\|_{\infty}+\kappa_{1}\left\|u^{\prime}\right\|_{\infty}+\kappa_{2}\left\|u^{\prime \prime}\right\|_{\left[\frac{\eta}{\alpha}, \eta\right]}\right) \int_{\frac{\eta}{\alpha}}^{\eta} \kappa_{0} g_{0}(s) d s \\
& =\lambda\left(f_{0}-\varepsilon\right)\left(\kappa_{0}\|u\|_{\infty}+\kappa_{1}\left\|u^{\prime}\right\|_{\infty}+\kappa_{2}\left\|u^{\prime \prime}\right\|_{\left[\frac{\eta}{\alpha}, \eta\right]}\right) \wedge_{4} \geqslant \lambda\left(f_{0}-\varepsilon\right) \Lambda_{4} \kappa_{0} u(t), \quad \forall t \in\left[\frac{\eta}{\alpha}, \eta\right],
\end{aligned}
$$

and

$$
\begin{aligned}
(T u)^{\prime}(t) & =\lambda \int_{0}^{1} \frac{\partial G}{\partial t}(t, s) f\left(s, u(s), u^{\prime}(s), u^{\prime \prime}(s)\right) d s \geqslant \lambda \int_{0}^{1} k_{1} g_{1}(s) f\left(s, u(s), u^{\prime}(s), u^{\prime \prime}(s)\right) d s \\
& \geqslant \lambda \int_{\frac{\eta}{\alpha}}^{\eta} k_{1} g_{1}(s) f\left(s, u(s), u^{\prime}(s), u^{\prime \prime}(s)\right) d s \\
& >\lambda \int_{\frac{\eta}{\alpha}}^{\eta} k_{1} g_{1}(s)\left(f_{0}-\varepsilon\right)\left(|u(s)|+\left|u^{\prime}(s)\right|+\left|u^{\prime \prime}(s)\right|\right) d s \\
& \geqslant \lambda\left(f_{0}-\varepsilon\right)\left(\kappa_{0}\|u\|_{\infty}+k_{1}\left\|u^{\prime}\right\|_{\infty}+\kappa_{2}\left\|u^{\prime \prime}\right\|_{\left[\frac{\eta}{\alpha}, \eta\right]}\right) \int_{\frac{\eta}{\alpha}}^{\eta} \kappa_{1} g_{1}(s) d s \\
& =\lambda\left(f_{0}-\varepsilon\right)\left(\kappa_{0}\|u\|_{\infty}+k_{1}\left\|u^{\prime}\right\|_{\infty}+\kappa_{2}\left\|u^{\prime \prime}\right\|_{\left[\frac{\eta}{\alpha}, \eta\right]}\right) \Lambda_{5} \geqslant \lambda\left(f_{0}-\varepsilon\right) \Lambda_{5} \kappa_{1} u^{\prime}(t), \quad \forall t \in\left[\frac{\eta}{\alpha}, \eta\right] .
\end{aligned}
$$

As a consequence we have that either $T u(t)>u(t)$ for all $t \in\left[\frac{\eta}{\alpha}, \eta\right]$ or $(T u)^{\prime}(t)>u^{\prime}(t)$ for all $t \in\left[\frac{\eta}{\alpha}, \eta\right]$, and so it is proved that $T u \npreceq u$. We deduce (see [7, Theorem 2.3.3]) that

$$
i_{K}\left(T, \Omega_{\delta_{1}}\right)=0 .
$$

On the other hand, due to the definition of $f^{\infty}$, we know that there exists $\tilde{\delta}_{2}>0$ such that when $\min \left\{|\mathbf{u}(\mathrm{t})|,\left|\mathbf{u}^{\prime}(\mathrm{t})\right|,\left|\mathbf{u}^{\prime \prime}(\mathrm{t})\right|\right\} \geqslant \tilde{\delta}_{2}$,

$$
f\left(t, u(t), u^{\prime}(t), u^{\prime \prime}(t)\right) \leqslant\left(f^{\infty}+\varepsilon\right)\left(|u(t)|+\left|u^{\prime}(t)\right|+\left|u^{\prime \prime}(t)\right|\right) \leqslant 3\left(f^{\infty}+\varepsilon\right)\|u\|, \quad \forall t \in[0,1] .
$$

Let $\delta_{2}>\left\{\delta_{1}, \tilde{\delta}_{2}\right\}$ and define

$$
\Omega_{\delta_{2}}=\left\{u \in K ; \min _{t \in[0,1]}|u(t)|<\delta_{2}\right\} \cup\left\{u \in K ; \min _{t \in[0,1]}\left|u^{\prime}(t)\right|<\delta_{2}\right\} \cup\left\{u \in K ; \min _{t \in[0,1]}\left|u^{\prime \prime}(t)\right|<\delta_{2}\right\} .
$$


We note that $\Omega_{\delta_{2}}$ is an unbounded subset of the cone K. Because of this, the fixed point index of operator T with respect to $\Omega_{\delta_{2}}, i_{K}\left(T, \Omega_{\delta_{2}}\right)$, is only defined in the case that the set of fixed points of operator $T$ in $\Omega_{\delta_{2}}$, that is, (I-T) ${ }^{-1}(\{0\}) \cap \Omega_{\delta_{2}}$, is compact (see [5] for the details). We will see that $i_{K}\left(T, \Omega_{\delta_{2}}\right)$ can be defined in this case.

First of all, since $(I-T)$ is a continuous operator, it is obvious that $(I-T)^{-1}(\{0\}) \cap \Omega_{\delta_{2}}$ is closed.

Moreover, we can assume that $(\mathrm{I}-\mathrm{T})^{-1}(\{0\}) \cap \Omega_{\delta_{2}}$ is bounded. Indeed, on the contrary, we would have infinite fixed points of operator $\mathrm{T}$ on $\Omega_{\delta_{2}}$ and it would be immediately deduced that problem (1.1)-(1.2) has an infinite number of positive solutions. Therefore we may assume that there exists a constant $M>0$ such that $\|\mathrm{u}\|<M$ for all $u \in(I-T)^{-1}(\{0\}) \cap \Omega_{\delta_{2}}$.

Finally, we will see that $(I-T)^{-1}(\{0\}) \cap \Omega_{\delta_{2}}$ is equicontinuous. This property follows from the fact that (I-T $)^{-1}(\{0\}) \cap \Omega_{\delta_{2}}$ is bounded. The proof is totally analogous to Step 2 in the proof of Lemma 3.1.

Now, we will calculate $i_{K}\left(T, \Omega_{\delta_{2}}\right)$. In particular, we will prove that $\|T u\| \leqslant\|u\|$ for all $u \in \partial \Omega_{\delta_{2}}$. Let $u \in \partial \Omega_{\delta_{2}}$, that is, $u \in K$ such that

$$
\min \left\{\min _{t \in[0,1]}|u(t)|, \min _{t \in[0,1]}\left|u^{\prime}(t)\right|, \min _{t \in[0,1]}\left|u^{\prime \prime}(t)\right|\right\}=\delta_{2} .
$$

Then,

$$
\begin{aligned}
|T u(t)| & =T u(t)=\lambda \int_{0}^{1} G(t, s) f\left(s, u(s), u^{\prime}(s), u^{\prime \prime}(s)\right) d s \\
& \leqslant \lambda \int_{0}^{1} g_{0}(s) f\left(s, u(s), u^{\prime}(s), u^{\prime \prime}(s)\right) d s \leqslant 3 \lambda \int_{0}^{1} g_{0}(s)\left(f^{\infty}+\varepsilon\right)\|u\| d s \\
& =3 \lambda\left(f^{\infty}+\varepsilon\right)\|u\| \Lambda_{1} \leqslant \lambda\left(f^{\infty}+\varepsilon\right)\|u\| \bar{\Lambda} \leqslant\|u\|, \\
\left|(T u)^{\prime}(t)\right| & =(T u)^{\prime}(t)=\lambda \int_{0}^{1} \frac{\partial G}{\partial t}(t, s) f\left(s, u(s), u^{\prime}(s), u^{\prime \prime}(s)\right) d s \\
& \leqslant \lambda \int_{0}^{1} g_{1}(s) f\left(s, u(s), u^{\prime}(s), u^{\prime \prime}(s)\right) d s \leqslant 3 \lambda \int_{0}^{1} g_{1}(s)\left(f^{\infty}+\varepsilon\right)\|u\| d s \\
& =3 \lambda\left(f^{\infty}+\varepsilon\right)\|u\| \Lambda_{2} \leqslant \lambda\left(f^{\infty}+\varepsilon\right)\|u\| \bar{\Lambda} \leqslant\|u\|,
\end{aligned}
$$

and

$$
\begin{aligned}
\left|(T u)^{\prime \prime}(t)\right| & \leqslant \lambda \int_{0}^{1}\left|\frac{\partial^{2} G}{\partial t^{2}}(t, s)\right| f\left(s, u(s), u^{\prime}(s), u^{\prime \prime}(s)\right) d s \\
& \leqslant \lambda \int_{0}^{1} \max \left\{g_{2}(s), \frac{1-\eta}{1-\alpha \eta}\right\} f\left(s, u(s), u^{\prime}(s), u^{\prime \prime}(s)\right) d s \\
& \leqslant 3 \lambda \int_{0}^{1} \max \left\{g_{2}(s), \frac{1-\eta}{1-\alpha \eta}\right\}\left(f^{\infty}+\varepsilon\right)\|u\| d s \\
& =3 \lambda\left(f^{\infty}+\varepsilon\right)\|u\| \Lambda_{3} \leqslant \lambda\left(f^{\infty}+\varepsilon\right)\|u\| \bar{\Lambda} \leqslant\|u\| .
\end{aligned}
$$

We deduce that

$$
\|\mathrm{Tu}\| \leqslant\|u\|,
$$

and as a consequence (see [5, Corollary 7.4]) we have that

$$
i_{K}\left(T, \Omega_{\delta_{2}}\right)=1 .
$$

Then, we conclude that $T$ has a fixed point in $\bar{\Omega}_{\delta_{2}} \backslash \Omega_{\delta_{1}}$, that is, there exists at least a positive solution for problem (1.1)-(1.2). 
Consequently, we obtain the following results.

Corollary 3.3. Assume that hypotheses (H1)-(H3) hold. Then,

(i) If $\mathrm{f}_{0}=\infty$ and $\mathrm{f}^{\infty}=0$, then for all $\lambda \in(0, \infty)$, problem (1.1)-(1.2) has at least a positive solution.

(ii) If $\mathrm{f}_{0}=\infty$ and $0<\mathrm{f}^{\infty}<\infty$, then for all $\lambda \in\left(0, \frac{1}{\bar{\Lambda} \mathrm{f}^{\infty}}\right)$, problem (1.1)-(1.2) has at least a positive solution.

(iii) If $0<\mathrm{f}_{0}<\infty$ and $\mathrm{f}^{\infty}=0$, then for all $\lambda \in\left(\frac{1}{\underline{\Lambda} \mathrm{f}_{0}}, \infty\right)$, problem (1.1)-(1.2) has at least a positive solution.

Remark 3.4. For the sake of completeness, we will give the exact expression of $\Lambda_{i}, i=1, \cdots, 5$ :

$$
\begin{aligned}
\Lambda_{1}= & \frac{\alpha+1}{6(1-\alpha \eta)}, \\
\Lambda_{2}= & \frac{1}{2(1-\alpha \eta)^{\prime}}, \\
\Lambda_{3}= & \frac{\alpha^{2}-2 \alpha\left(\alpha^{2}+1\right) \eta+\left(\alpha^{4}+3 \alpha^{3}+\alpha+1\right) \eta^{2}-2(\alpha(\alpha(\alpha(2 \alpha-3)+5)-3)+1) \eta^{3}}{2(\alpha-1) \eta(\alpha \eta-1)(\alpha((\alpha-1) \eta-1)+\eta)} \\
& +\frac{\alpha^{2}(\alpha((\alpha-2) \alpha+3)-1) \eta^{4}}{2(\alpha-1) \eta(\alpha \eta-1)(\alpha((\alpha-1) \eta-1)+\eta)^{\prime}}, \\
\Lambda_{4}= & \frac{\eta^{4}\left(\alpha^{3}(2 \eta-3)+3 \alpha-2 \eta\right)}{12 \alpha^{5}(\alpha \eta-1)} \min \{\alpha-1,1\}, \\
\Lambda_{5}= & \frac{(\alpha-1) \eta^{2}(\alpha(\eta-2)+\eta)}{2 \alpha^{2}(\alpha \eta-1)} .
\end{aligned}
$$

\section{Existence and multiplicity of solutions}

In this section we will give some conditions to ensure the existence of multiple solutions of the boundary problem (1.1)-(1.2). To do that, we will use the fixed point index theory. Similar arguments have been applied in [2] to functional equations that only depend on the values of the solution $u$. First of all, we will compile some classical results regarding to this theory (see [1, 7] for more details).

Lemma 4.1. Let $\mathrm{D}$ be an open bounded set with $\mathrm{D}_{\mathrm{K}}=\mathrm{D} \cap \mathrm{K} \neq \emptyset$ and $\overline{\mathrm{D}}_{\mathrm{K}} \neq \mathrm{K}$. Assume that $\mathrm{F}: \overline{\mathrm{D}}_{\mathrm{K}} \rightarrow \mathrm{K}$ is a compact map such that $\mathrm{x} \neq \mathrm{F} x$ for $\mathrm{x} \in \partial \mathrm{D}_{\mathrm{K}}$. Then the fixed point index $i_{\mathrm{K}}\left(\mathrm{F}, \mathrm{D}_{\mathrm{K}}\right)$ satisfies the following properties:

(1) If there exists $e \in K \backslash\{0\}$ such that $x \neq F x+\alpha$ for all $x \in \partial D_{K}$ and all $\alpha>0$, then $i_{K}\left(F, D_{K}\right)=0$.

(2) If $\mu x \neq \mathrm{F} x$ for all $\mathrm{x} \in \partial \mathrm{D}_{\mathrm{K}}$ and for every $\mu \geqslant 1$, then $i_{\mathrm{K}}\left(\mathrm{F}, \mathrm{D}_{\mathrm{K}}\right)=1$.

(3) Let $\mathrm{D}^{1}$ be open in $\mathrm{X}$ with $\overline{\mathrm{D}}^{1} \subset \mathrm{D}_{\mathrm{K}}$. If $i_{\mathrm{K}}\left(\mathrm{F}, \mathrm{D}_{\mathrm{K}}\right)=1$ and $i_{\mathrm{K}}\left(\mathrm{F}, \mathrm{D}_{\mathrm{K}}^{1}\right)=0$, then $\mathrm{F}$ has a fixed point in $\mathrm{D}_{\mathrm{K}} \backslash \overline{\mathrm{D}}_{\mathrm{K}}^{1}$. The same result holds if $i_{\mathrm{K}}\left(\mathrm{F}, \mathrm{D}_{\mathrm{K}}\right)=0$ and $i_{\mathrm{K}}\left(\mathrm{F}, \mathrm{D}_{\mathrm{K}}^{1}\right)=1$.

We will consider the following sets:

$$
\begin{gathered}
K_{\rho}=\{u \in K ;\|u\|<\rho\}, \\
V_{\rho}=\left\{u \in K ; \min _{t \in\left[\frac{\eta}{\alpha}, \eta\right]} u(t)<\rho, \min _{t \in\left[\frac{\eta}{\alpha}, \eta\right]} u^{\prime}(t)<\rho,\left\|u^{\prime \prime}\right\|_{\infty}<\rho\right\} .
\end{gathered}
$$

It is clear that

$$
\mathrm{K}_{\rho} \subset \mathrm{V}_{\rho} \subset \mathrm{K}_{\frac{\rho}{\mathrm{c}}}
$$

where $c=\min \left\{\kappa_{0}, \kappa_{1}, \kappa_{2}\right\}$. 0 .

In the two following lemmas we give some sufficient conditions to ensure that the index is either 1 or 
Lemma 4.2. Let (see Remark 4.5)

$$
\frac{1}{m}=\max \left\{\sup _{t \in[0,1]} \int_{0}^{1} G(t, s) d s, \sup _{t \in[0,1]} \int_{0}^{1} \frac{\partial G}{\partial t}(t, s) d s, \sup _{t \in[0,1]} \int_{0}^{1}\left|\frac{\partial^{2} G}{\partial t^{2}}(t, s)\right| d s\right\},
$$

and

$$
f^{\rho}=\sup \left\{\frac{f(t, u, v, w)}{\rho} ;(t, u, v, w) \in[0,1] \times[0, \rho] \times[0, \rho] \times[-\rho, \rho]\right\} .
$$

If there exists $\rho>0$ such that

$$
\lambda \frac{f^{\rho}}{m}<1
$$

then $i_{K}\left(T, K_{\rho}\right)=1$.

Proof. We will prove that $T u \neq \mu u$ for all $u \in \partial K_{\rho}$ and for every $\mu \geqslant 1$.

Suppose, on the contrary, that there exist some $u \in \partial K_{\rho}$ and $\mu \geqslant 1$ such that

$$
\mu u(t)=\lambda \int_{0}^{1} G(t, s) f\left(s, u(s), u^{\prime}(s), u^{\prime \prime}(s)\right) d s .
$$

Taking the supremum for $t \in[0,1]$, we obtain that

$$
\mu\|u\|_{\infty}=\lambda \sup _{t \in[0,1]} \int_{0}^{1} G(t, s) f\left(s, u(s), u^{\prime}(s), u^{\prime \prime}(s)\right) d s \leqslant \lambda \rho f^{\rho} \sup _{t \in[0,1]} \int_{0}^{1} G(t, s) d s \leqslant \lambda \rho \frac{f^{\rho}}{m}<\rho .
$$

On the other hand, we have that

$$
\mu u^{\prime}(t)=\lambda \int_{0}^{1} \frac{\partial G}{\partial t}(t, s) f\left(s, u(s), u^{\prime}(s), u^{\prime \prime}(s)\right) d s,
$$

and so

$$
\mu\left\|u^{\prime}\right\|_{\infty}=\lambda \sup _{t \in[0,1]} \int_{0}^{1} \frac{\partial G}{\partial t}(t, s) f\left(s, u(s), u^{\prime}(s), u^{\prime \prime}(s)\right) d s \leqslant \lambda \rho f^{\rho} \sup _{t \in[0,1]} \int_{0}^{1} \frac{\partial G}{\partial t}(t, s) d s \leqslant \lambda \rho \frac{f^{\rho}}{m}<\rho .
$$

Finally, it holds that

$$
\mu u^{\prime \prime}(t)=\lambda \int_{0}^{1} \frac{\partial^{2} G}{\partial t^{2}}(t, s) f\left(s, u(s), u^{\prime}(s), u^{\prime \prime}(s)\right) d s
$$

and so

$$
\mu\left\|u^{\prime \prime}\right\|_{\infty} \leqslant \lambda \sup _{t \in[0,1]} \int_{0}^{1}\left|\frac{\partial^{2} G}{\partial t^{2}}(t, s)\right| f\left(s, u(s), u^{\prime}(s), u^{\prime \prime}(s)\right) d s \leqslant \lambda \rho f^{\rho} \sup _{t \in[0,1]} \int_{0}^{1}\left|\frac{\partial^{2} G}{\partial t^{2}}(t, s)\right| d s \leqslant \lambda \rho \frac{f^{\rho}}{m}<\rho .
$$

As a consequence, it can be deduced that

$$
\mu \rho=\mu \max \left\{\|u\|_{\infty},\left\|u^{\prime}\right\|_{\infty},\left\|u^{\prime \prime}\right\|_{\infty}\right\}<\rho,
$$

which is a contradiction with the assumption that $\mu \geqslant 1$. Therefore, the result is proved.

Lemma 4.3. Let (see Remark 4.5)

$$
\frac{1}{M}=\max \left\{\inf _{t \in\left[\frac{\eta}{\alpha}, \eta\right]} \int_{\frac{\eta}{\alpha}}^{\eta} G(t, s) d s, \inf _{t \in\left[\frac{\eta}{\alpha}, \eta\right]} \int_{\frac{\eta}{\alpha}}^{\eta} \frac{\partial G}{\partial t}(t, s) d s, \inf _{t \in\left[\frac{\eta}{\alpha}, \eta\right]} \int_{\frac{\eta}{\alpha}}^{\eta} \frac{\partial^{2} G}{\partial t^{2}}(t, s) d s\right\},
$$


and

$$
f_{\rho}=\inf \left\{\frac{f(t, u, v, w)}{\rho} ;(t, u, v, w) \in\left[\frac{\eta}{\alpha}, \eta\right] \times\left[0, \frac{\rho}{k_{0}}\right] \times\left[0, \frac{\rho}{\mathrm{k}_{1}}\right] \times[0, \rho]\right\} .
$$

If there exists $\rho>0$ such that

$$
\lambda \frac{f_{\rho}}{M}>1
$$

then $i_{K}\left(T, V_{\rho}\right)=0$.

Proof. We will prove that there exists $e \in K \backslash\{0\}$ such that $u \neq T u+\alpha e$ for all $u \in \partial V_{\rho}$ and all $\alpha>0$.

Let us take $e(t)=1$ and suppose that there exist some $u \in \partial V_{\rho}$ and $\alpha>0$ such that $u=T u+\alpha$. Then,

$$
\begin{aligned}
u(t) & =\lambda \int_{0}^{1} G(t, s) f\left(s, u(s), u^{\prime}(s), u^{\prime \prime}(s)\right) d s+\alpha>\lambda \int_{0}^{1} G(t, s) f\left(s, u(s), u^{\prime}(s), u^{\prime \prime}(s)\right) d s \\
& \geqslant \lambda \int_{\frac{\eta}{\alpha}}^{\eta} G(t, s) f\left(s, u(s), u^{\prime}(s), u^{\prime \prime}(s)\right) d s \geqslant \lambda \rho f_{\rho} \int_{\frac{\eta}{\alpha}}^{\eta} G(t, s) d s, \\
u^{\prime}(t) & =\lambda \int_{0}^{1} \frac{\partial G}{\partial t}(t, s) f\left(s, u(s), u^{\prime}(s), u^{\prime \prime}(s)\right) d s \geqslant \lambda \int_{\frac{\eta}{\alpha}}^{\eta} \frac{\partial G}{\partial t}(t, s) f\left(s, u(s), u^{\prime}(s), u^{\prime \prime}(s)\right) d s \\
& \geqslant \lambda \rho f_{\rho} \int_{\frac{\eta}{\alpha}}^{\eta} \frac{\partial G}{\partial t}(t, s) d s,
\end{aligned}
$$

and for $\mathrm{t} \in\left[\frac{\eta}{\alpha}, \eta\right]$,

$$
\begin{aligned}
u^{\prime \prime}(t) & =\lambda \int_{0}^{1} \frac{\partial^{2} G}{\partial t^{2}}(t, s) f\left(s, u(s), u^{\prime}(s), u^{\prime \prime}(s)\right) d s \geqslant \lambda \int_{\frac{\eta}{\alpha}}^{\eta} \frac{\partial^{2} G}{\partial t^{2}}(t, s) f\left(s, u(s), u^{\prime}(s), u^{\prime \prime}(s)\right) d s \\
& \geqslant \lambda \rho f_{\rho} \int_{\frac{\eta}{\alpha}}^{\eta} \frac{\partial^{2} G}{\partial t^{2}}(t, s) d s .
\end{aligned}
$$

Consequently, either $u(t)>\rho, u^{\prime}(t)>\rho$ or $u^{\prime \prime}(t)>\rho$ for $t \in\left[\frac{\eta}{\alpha}, \eta\right]$, which is a contradiction. Therefore, we conclude the veracity of the result.

From the previous lemmas, it is possible to formulate the following theorem, in which we give some conditions under which problem (3.1) has multiple solutions. In this case, we establish conditions to ensure the existence of one, two or three solutions. However, it must be pointed out that similar results can be formulated to ensure the existence of four or more solutions.

Theorem 4.4. The integral equation (3.1) has at least one nontrivial solution in $\mathrm{K}$ if one of the following conditions holds:

(C1) There exist $\rho_{1}, \rho_{2} \in(0, \infty), \frac{\rho_{1}}{c}<\rho_{2}$, such that $\left(\mathrm{I}_{\rho_{1}}^{0}\right)$ and $\left(\mathrm{I}_{\rho_{2}}^{1}\right)$ are satisfied.

(C2) There exist $\rho_{1}, \rho_{2} \in(0, \infty), \rho_{1}<\rho_{2}$, such that $\left(\mathrm{I}_{\rho_{1}}^{1}\right)$ and $\left(\mathrm{I}_{\rho_{2}}^{0}\right)$ are satisfied.

The integral equation (3.1) has at least two nontrivial solutions in $\mathrm{K}$ if one of the following conditions holds:

(C3) There exist $\rho_{1}, \rho_{2}, \rho_{3} \in(0, \infty), \frac{\rho_{1}}{\mathrm{c}}<\rho_{2}<\rho_{3}$, such that $\left(\mathrm{I}_{\rho_{1}}^{0}\right),\left(\mathrm{I}_{\rho_{2}}^{1}\right)$ and $\left(\mathrm{I}_{\rho_{3}}^{0}\right)$ are satisfied.

(C4) There exist $\rho_{1}, \rho_{2}, \rho_{3} \in(0, \infty)$, with $\rho_{1}<\rho_{2}$ and $\frac{\rho_{2}}{\mathrm{c}}<\rho_{3}$, such that $\left(\mathrm{I}_{\rho_{1}}^{1}\right),\left(\mathrm{I}_{\rho_{2}}^{0}\right)$ and $\left(\mathrm{I}_{\rho_{3}}^{1}\right)$ are satisfied.

The integral equation (3.1) has at least three nontrivial solutions in $\mathrm{K}$ if one of the following conditions holds:

(C5) There exist $\rho_{1}, \rho_{2}, \rho_{3}, \rho_{4} \in(0, \infty)$, with $\frac{\rho_{1}}{\mathrm{c}}<\rho_{2}<\rho_{3}$ and $\frac{\rho_{3}}{\mathrm{c}}<\rho_{4}$, such that $\left(\mathrm{I}_{\rho_{1}}^{0}\right),\left(\mathrm{I}_{\rho_{2}}^{1}\right),\left(\mathrm{I}_{\rho_{3}}^{0}\right)$ and $\left(\mathrm{I}_{\rho_{4}}^{1}\right)$ are satisfied. 
(C6) There exist $\rho_{1}, \rho_{2}, \rho_{3}, \rho_{4} \in(0, \infty)$, with $\rho_{1}<\rho_{2}$ and $\frac{\rho_{2}}{c}<\rho_{3}<\rho_{4}$, such that $\left(\mathrm{I}_{\rho_{1}}^{1}\right),\left(\mathrm{I}_{\rho_{2}}^{0}\right),\left(\mathrm{I}_{\rho_{3}}^{1}\right)$ and $\left(\mathrm{I}_{\rho_{4}}^{0}\right)$ are satisfied. index.

The proof of the previous result is an immediate consequence of the properties of the fixed point

Remark 4.5. For the sake of completeness, we give the exact expression of the components involved in the formulas of $\frac{1}{\mathrm{~m}}$ and $\frac{1}{\mathrm{M}}$ :

$$
\int_{0}^{1} G(t, s) d s=\frac{1}{12} t^{2}\left(\frac{3\left(\alpha \eta^{2}-1\right)}{\alpha \eta-1}-2 t\right),
$$

and

$$
\sup _{t \in[0,1]} \int_{0}^{1} G(t, s) d s=\int_{0}^{1} G(1, s) d s=\frac{\alpha \eta(2-3 \eta)+1}{12(1-\alpha \eta)} .
$$

Moreover,

$$
\int_{0}^{1} \frac{\partial G}{\partial t}(t, s) d s=\frac{t(\alpha \eta(\eta-t)+t-1)}{2(\alpha \eta-1)}
$$

and

$$
\sup _{t \in[0,1]} \int_{0}^{1} \frac{\partial G}{\partial t}(t, s) d s=\int_{0}^{1} \frac{\partial G}{\partial t}(1, s) d s=\frac{\alpha \eta(1-\eta)}{2(1-\alpha \eta)}
$$

Finally,

$$
\int_{0}^{1} \frac{\partial^{2} G}{\partial t^{2}}(t, s) d s= \begin{cases}\frac{\alpha \eta(\eta-2 t)+2 t-1}{2(\alpha \eta-1)}, & t \leqslant \alpha \eta \\ \frac{-2 \alpha^{2} \eta^{2}+\alpha \eta(\eta+2 t)-2(t-1) t-1}{2(\alpha \eta-1)}, & t>\alpha \eta\end{cases}
$$

and

$$
\sup _{t \in[0,1]} \int_{0}^{1} \frac{\partial^{2} G}{\partial t^{2}}(t, s) d s=\int_{0}^{1} \frac{\partial^{2} G}{\partial t^{2}}(0, s) d s=\frac{1-\alpha \eta^{2}}{2(1-\alpha \eta)} .
$$

Now, it is easy to verify that

$$
\frac{1}{m}=\max \left\{\frac{\alpha \eta(2-3 \eta)+1}{12(1-\alpha \eta)}, \frac{\alpha \eta(1-\eta)}{2(1-\alpha \eta)}, \frac{1-\alpha \eta^{2}}{2(1-\alpha \eta)}\right\}=\frac{1-\alpha \eta^{2}}{2(1-\alpha \eta)} .
$$

On the other hand, for $t \in\left[\frac{\eta}{\alpha}, \eta\right]$,

$$
\int_{\frac{\eta}{\alpha}}^{\eta} G(t, s) d s=\frac{1}{12}\left(\frac{2 \eta^{3}}{\alpha^{3}}-\frac{6 \eta^{2} t}{\alpha^{2}}+\frac{3 \eta t^{2}(\alpha(\alpha(\alpha \eta+\eta-2)+\eta)-\eta)}{\alpha^{2}(\alpha \eta-1)}-2 t^{3}\right),
$$

and

$$
\inf _{t \in\left[\frac{\eta}{\alpha}, \eta\right]} \int_{\frac{\eta}{\alpha}}^{\eta} G(t, s) d s=\int_{\frac{\eta}{\alpha}}^{\eta} G\left(\frac{\eta}{\alpha}, s\right) d s=\frac{(\alpha-1) \eta^{3}(\alpha(2-\alpha \eta)-\eta)}{4 \alpha^{4}(1-\alpha \eta)} .
$$

In addition,

$$
\int_{\frac{\eta}{\alpha}}^{\eta} \frac{\partial G}{\partial t}(t, s) d s=\frac{-\alpha^{2} \eta t(\alpha t+2)+\alpha^{2} t^{2}+\eta^{2}\left(\left(\alpha^{3}+\alpha^{2}+\alpha-1\right) t+1\right)-\alpha \eta^{3}}{2 \alpha^{2}(\alpha \eta-1)}
$$

and

$$
\inf _{t \in\left[\frac{\eta}{\alpha}, \eta\right]} \int_{\frac{\eta}{\alpha}}^{\eta} \frac{\partial G}{\partial t}(t, s) d s=\int_{\frac{\eta}{\alpha}}^{\eta} \frac{\partial G}{\partial t}\left(\frac{\eta}{\alpha}, s\right) d s=\frac{(\alpha-1) \eta^{2}(\alpha(2-\alpha \eta)-\eta)}{2 \alpha^{3}(1-\alpha \eta)}
$$


Finally,

$$
\int_{\frac{\eta}{\alpha}}^{\eta} \frac{\partial^{2} G}{\partial t^{2}}(t, s) d s=\frac{\eta(\alpha(\alpha(\alpha \eta+\eta-2)+\eta)-\eta)}{2 \alpha^{2}(\alpha \eta-1)}-t
$$

and

$$
\inf _{t \in\left[\frac{\eta}{\alpha}, \eta\right]} \int_{\frac{\eta}{\alpha}}^{\eta} \frac{\partial^{2} G}{\partial t^{2}}(t, s) d s=\int_{\frac{\eta}{\alpha}}^{\eta} \frac{\partial^{2} G}{\partial t^{2}}(\eta, s) d s=\frac{(\alpha-1)^{2}(\alpha+1) \eta^{2}}{2 \alpha^{2}(1-\alpha \eta)}
$$

Now we can calculate

$$
\begin{aligned}
\frac{1}{M} & =\max \left\{\frac{(\alpha-1) \eta^{3}(\alpha(2-\alpha \eta)-\eta)}{4 \alpha^{4}(1-\alpha \eta)}, \frac{(\alpha-1) \eta^{2}(\alpha(2-\alpha \eta)-\eta)}{2 \alpha^{3}(1-\alpha \eta)}, \frac{(\alpha-1)^{2}(\alpha+1) \eta^{2}}{2 \alpha^{2}(1-\alpha \eta)}\right\} \\
& =\frac{(\alpha-1) \eta^{2}(\alpha(2-\alpha \eta)-\eta)}{2 \alpha^{3}(1-\alpha \eta)} .
\end{aligned}
$$

\section{Results of non-existence of solution}

If the following theorem we give some conditions to ensure that the integral equation (3.1) has not nontrivial solution in $\mathrm{K}$.

Theorem 5.1. If one of the following conditions holds

(i) $f(t, x, y, z)<\tilde{m} \max \{x, y,|z|\}$ for every $t \in[0,1], x, y \geqslant 0$ and $z \in \mathbb{R}$, where

$$
\frac{1}{\tilde{m}}=\max \left\{\sup _{t \in[0,1]} \lambda \int_{0}^{1} G(t, s) d s, \sup _{t \in[0,1]} \lambda \int_{0}^{1} \frac{\partial G}{\partial t}(t, s) d s, \sup _{t \in[0,1]} \lambda \int_{0}^{1}\left|\frac{\partial^{2} G}{\partial t^{2}}(t, s)\right| d s\right\} ;
$$

(ii) $f(t, x, y, z)>M x$ for every $t \in[a, b] \subset\left[\frac{\eta}{\alpha}, \eta\right]$, with $a \neq b, x, y \geqslant 0$ and $z \in \mathbb{R}$, where

$$
\frac{1}{M}=\inf _{t \in[a, b]} \lambda \int_{a}^{b} G(t, s) d s
$$

(iii) $f(t, x, y, z)>\tilde{M} y$ for every $t \in[a, b] \subset\left[\frac{\eta}{\alpha}, \eta\right]$, with $a \neq b, x, y \geqslant 0$ and $z \in \mathbb{R}$, where

$$
\frac{1}{\tilde{M}}=\inf _{t \in[a, b]} \lambda \int_{a}^{b} \frac{\partial G}{\partial t}(t, s) d s
$$

then the integral equation (3.1) has not nontrivial solution in $\mathrm{K}$.

Proof. We will only prove (i) and (ii) since item (iii) is totally analogous to (ii).

(i) Suppose, on the contrary, that there exists $u \in K$ such that $u=T u$. Let $t_{0} \in[0,1]$ be such that $\|u\|_{\infty}=u\left(t_{0}\right)$. Then,

$$
\begin{aligned}
\|u\|_{\infty} & =\lambda \int_{0}^{1} G\left(t_{0}, s\right) f\left(s, u(s), u^{\prime}(s), u^{\prime \prime}(s)\right) d s \\
& <\lambda \int_{0}^{1} G\left(t_{0}, s\right) \tilde{m} \max \left\{u(s), u^{\prime}(s),\left|u^{\prime \prime}(s)\right|\right\} d s \\
& \leqslant \lambda \tilde{m}\|u\| \int_{0}^{1} G\left(t_{0}, s\right) d s \leqslant\|u\| .
\end{aligned}
$$

Now, let $t_{1} \in[0,1]$ such that $\left\|u^{\prime}\right\|_{\infty}=u^{\prime}\left(t_{1}\right)$. Then,

$$
\left\|u^{\prime}\right\|_{\infty}=\lambda \int_{0}^{1} \frac{\partial G}{\partial t}\left(t_{1}, s\right) f\left(s, u(s), u^{\prime}(s), u^{\prime \prime}(s)\right) d s
$$




$$
\begin{aligned}
& <\lambda\|\mathfrak{u}\| \int_{0}^{1} \frac{\partial G}{\partial t}\left(t_{1}, s\right) \tilde{m} \max \left\{u(s), u^{\prime}(s),\left|u^{\prime \prime}(s)\right|\right\} d s \\
& \leqslant \lambda \tilde{m}\|u\| \int_{0}^{1} \frac{\partial G}{\partial t}\left(t_{1}, s\right) d s \leqslant\|u\| .
\end{aligned}
$$

Finally, let $t_{2} \in[0,1]$ such that $\left\|u^{\prime \prime}\right\|_{\infty}=\left|u^{\prime \prime}\left(t_{2}\right)\right|$. Then,

$$
\begin{aligned}
\left\|u^{\prime \prime}\right\|_{\infty} & =\left|\lambda \int_{0}^{1} \frac{\partial^{2} G}{\partial t^{2}}\left(t_{2}, s\right) f\left(s, u(s), u^{\prime}(s), u^{\prime \prime}(s)\right) d s\right| \\
& \leqslant \lambda \int_{0}^{1}\left|\frac{\partial^{2} G}{\partial t^{2}}\left(t_{2}, s\right)\right| f\left(s, u(s), u^{\prime}(s), u^{\prime \prime}(s)\right) d s \\
& <\lambda \int_{0}^{1}\left|\frac{\partial^{2} G}{\partial t^{2}}\left(t_{2}, s\right)\right| \tilde{m} \max \left\{u(s), u^{\prime}(s),\left|u^{\prime \prime}(s)\right|\right\} d s \\
& \leqslant \lambda \tilde{m}\|u\| \int_{0}^{1}\left|\frac{\partial^{2} G}{\partial t^{2}}\left(t_{2}, s\right)\right| d s \leqslant\|u\| .
\end{aligned}
$$

Consequently, we reach to

$$
\|u\|=\max \left\{\|u\|_{\infty},\left\|u^{\prime}\right\|_{\infty}\left\|u^{\prime \prime}\right\|_{\infty}\right\}<\|u\|,
$$

which is a contradiction.

(ii) Suppose, on the contrary, that there exists $u \in K$ such that $u=T u$. Let $t_{0} \in[0,1]$ be such that $u\left(t_{0}\right)=\min _{t \in[a, b]} u(t)$. Then, for $t \in[a, b]$ we have that

$$
\begin{aligned}
u(t) & =\lambda \int_{0}^{1} G(t, s) f\left(s, u(s), u^{\prime}(s), u^{\prime \prime}(s)\right) d s \geqslant \lambda \int_{a}^{b} G(t, s) f\left(s, u(s), u^{\prime}(s), u^{\prime \prime}(s)\right) d s \\
& >M \lambda \int_{a}^{b} G(t, s) u(s) d s .
\end{aligned}
$$

Therefore, we arrive at

$$
u\left(t_{0}\right)=\min _{t \in[a, b]} u(t)>M \inf _{t \in[a, b]} \lambda \int_{a}^{b} G(t, s) u(s) d s \geqslant M u\left(t_{0}\right) \inf _{t \in[a, b]} \lambda \int_{a}^{b} G(t, s) d s=u\left(t_{0}\right),
$$

which is a contradiction.

\section{Examples}

In this section we will consider several examples which show that the existence results proved in Theorems 3.2 and 4.4 are not comparable.

Example 6.1. Let us consider the problem with $f(t, x, y, z)=\frac{h(t)}{x^{2}+y^{2}+z^{2}}$, where $c_{1} \geqslant h(t) \geqslant c_{2}>0$ for all $t \in[0,1]$, and $\eta$ and $\alpha$ arbitrarily chosen, that is,

$$
\left\{\begin{array}{l}
-u^{(3)}(t)=\lambda \frac{h(t)}{(u(t))^{2}+\left(u^{\prime}(t)\right)^{2}+\left(u^{\prime \prime}(t)\right)^{2}}, \quad t \in[0,1] \\
u(0)=u^{\prime}(0)=0, \quad u^{\prime}(1)=\alpha u^{\prime}(\eta) .
\end{array}\right.
$$

In this case,

$$
f_{0}=\lim _{|x|,|y|,|z| \rightarrow 0} \min _{t \in[0,1]} \frac{f(t, x, y, z)}{|x|+|y|+|z|}=\lim _{|x|,|y|,|z| \rightarrow 0} \frac{\min _{t \in[0,1]} h(t)}{\left(x^{2}+y^{2}+z^{2}\right)(|x|+|y|+|z|)}=+\infty,
$$


and

$$
f^{\infty}=\lim _{|x|,|y|,|z| \rightarrow \infty} \max _{t \in[0,1]} \frac{f(t, x, y, z)}{|x|+|y|+|z|}=\lim _{|x|,|y|,|z| \rightarrow \infty} \frac{\max _{t \in[0,1]} h(t)}{\left(x^{2}+y^{2}+z^{2}\right)(|x|+|y|+|z|)}=0,
$$

so Theorem 3.2 assures that there exists at least a positive solution of the problem for all $\lambda>0$.

On the other hand, let $\rho>0$. Then,

$$
f^{\rho}=\sup \left\{\frac{h(t)}{\rho\left(x^{2}+y^{2}+z^{2}\right)} ;(t, x, y, z) \in[0,1] \times[0, \rho] \times[0, \rho] \times[-\rho, \rho]\right\}=\infty,
$$

so it is not possible to find a positive $\rho$ such that $\lambda \frac{f^{\rho}}{m}<1$ and, consequently, Theorem 4.4 can not be applied in this case.

Example 6.2. Let us consider the problem with $f(t, x, y, z)=h(t)\left(x^{2}+y^{2}+z^{2}+1\right)$, where $c_{1} \geqslant h(t) \geqslant$ $c_{2}>0$ for all $t \in[0,1], \eta=\frac{1}{2}$ and $\alpha=\frac{3}{2}$, that is,

$$
\left\{\begin{array}{l}
-u^{(3)}(t)=\lambda h(t)\left((u(t))^{2}+\left(u^{\prime}(t)\right)^{2}+\left(u^{\prime \prime}(t)\right)^{2}+1\right), \quad t \in[0,1], \\
u(0)=u^{\prime}(0)=0, \quad u^{\prime}(1)=\frac{3}{2} u^{\prime}\left(\frac{1}{2}\right) .
\end{array}\right.
$$

In this case,

$$
f_{0}=\lim _{|x|,|y|,|z| \rightarrow 0} \min _{t \in[0,1]} \frac{f(t, x, y, z)}{|x|+|y|+|z|}=\lim _{|x|,|y|,|z| \rightarrow 0} \frac{\left(\min _{t \in[0,1]} h(t)\right)\left(x^{2}+y^{2}+z^{2}+1\right)}{|x|+|y|+|z|}=\infty,
$$

and

$$
f^{\infty}=\lim _{|x|,|y|,|z| \rightarrow \infty} \max _{t \in[0,1]} \frac{f(t, x, y, z)}{|x|+|y|+|z|}=\lim _{|x|,|y|,|z| \rightarrow \infty} \frac{\left(\max _{t \in[0,1]} h(t)\right)\left(x^{2}+y^{2}+z^{2}+1\right)}{|x|+|y|+|z|}=\infty,
$$

so Theorem 3.2 can not be applied.

However, we will see that Theorem 4.4 lets us ensure the existence of at least one positive solution for certain values of $\lambda$.

Let $\rho_{1}, \rho_{2}>0$. Then,

$$
f_{\rho_{1}}=\frac{1}{\rho_{1}} \inf _{t \in\left[\frac{1}{3}, \frac{1}{2}\right]} h(t)
$$

and

$$
f^{\rho_{2}}=\frac{1+3 \rho_{2}^{2}}{\rho_{2}} \sup _{t \in[0,1]} h(t)
$$

Moreover, $\frac{1}{m}=\frac{5}{4}$ and $\frac{1}{M}=\frac{11}{108}$. As a consequence of $(\mathrm{C} 1)$ in Theorem 4.4 , for any $\rho_{1}, \rho_{2}>0$ such that $\rho_{1}<c \rho_{2}=\frac{\rho_{2}}{90}$ and

$$
\frac{108 \rho_{1}}{11 \inf _{t \in\left[\frac{1}{3}, \frac{1}{2}\right]} h(t)}<\frac{4 \rho_{2}}{5\left(1+3 \rho_{2}^{2}\right) \sup _{t \in[0,1]} h(t)},
$$

there exists at least a nontrivial solution of problem (1.1)-(1.2) for all

$$
\lambda \in\left(\frac{108 \rho_{1}}{11 \inf _{t \in\left[\frac{1}{3}, \frac{1}{2}\right]} h(t)}, \frac{4 \rho_{2}}{5\left(1+3 \rho_{2}^{2}\right) \sup _{t \in[0,1]} h(t)}\right) .
$$

In particular, it can be deduced that there exists at least a nontrivial solution of problem (1.1)-(1.2) for all

$$
\lambda \in\left(0, \frac{2}{5 \sqrt{3} \sup _{t \in[0,1]} h(t)}\right) .
$$




\section{Acknowledgment}

First and second authors were partially supported by Ministerio de Economía y Competitividad, Spain, and FEDER, project MTM2013-43014-P, and by the Agencia Estatal de Investigación (AEI) of Spain under grant MTM2016-75140-P, co-financed by the European Community fund FEDER. Second author was partially supported by FPU scholarship, Ministerio de Educación, Cultura y Deporte, Spain. Third author was partially suported by National Founds through FCT-Fundação para a Ciência e a Tecnologia, Portugal, as part of project: SFRH/BSAB/114246/2016.

\section{References}

[1] H. Amann, Fixed point equations and nonlinear eigenvalue problems in ordered Banach spaces, SIAM. Rev., 18 (1976), 620-709. 4

[2] A. Cabada, G. Infante, F. A. F. Tojo, Nontrivial solutions of Hammerstein integral equations with reflections, Bound. Value Probl., 2013 (2013), 22 pages. 1, 4

[3] A. Cabada, F. Minhós, A. I. Santos, Solvability for a third order discontinuous fully equation with nonlinear functional boundary conditions, J. Math. Anal. Appl., 322 (2006), 735-748. 1

[4] J. Graef, L. Kong, F. Minhós, Generalized Hammerstein equations and applications, Results Math., 72 (2017), $369-383$. 1

[5] A. Granas, J. Dugundji, Fixed Point Theory, Springer, New York, (2003). 1, 3

[6] M. Greguš, Third Order Linear Differential Equations, Mathematics and its Applications, D. Reidel Publishing Co., Dordrecht, (1987). 1

[7] D. Guo, V. Lakshmikantham, Nonlinear Problems in Abstract Cones, Academic Press, New York, (1988). 1, 3, 4

[8] L. Guo, J. Sun, Y. Zhao, Existence of positive solutions for nonlinear third-order three-point boundary value problems, Nonlinear Anal., 68 (2008), 3151-3158. 1, 2, 2.1, 2.2

[9] G. Infante, P. Pietramala, A third order boundary value problem subject to nonlinear boundary conditions, Math. Bohem., 135 (2010), 113-121. 1

[10] G. Infante, P. Pietramala, F. A. F. Tojo, Nontrivial solutions of local and nonlocal Neumann boundary-value problems, Proc. Royal Soc. Edinburgh, 146 (2016), 337-369. 1

[11] G. Infante, J. R. L. Webb, Three point boundary value problems with solutions that change sign, J. Integral Equations Appl., 15 (2003), 37-57. 1

[12] Y. Liu, Z. Weiguo, L. Xiping, S. Chunfang, C. Hua, Positive solutions for a nonlinear third order multipoint boundary value problem, Pac. J. Math., 249 (2011), 177-188. 1

[13] F. Minhós, R. Sousa, On the solvability of third-order three point systems of differential equations with dependence on the first derivative, Bull. Braz. Math. Soc., 48 (2017), 485-503. 1, 2.3, 2.4 